\title{
Molecular mimicry of NMDA receptors may contribute to neuropsychiatric symptoms in severe COVID-19 cases
}

\author{
Veronika Vasilevska' ${ }^{1}$ Paul C. Guest ${ }^{2}$, Hans-Gert Bernstein ${ }^{1}$, Matthias L. Schroeter ${ }^{3}$, Christian Geis ${ }^{4,5}$ and \\ Johann Steiner ${ }^{1,5,6^{*}}$ (1)
}

\begin{abstract}
Approximately $30 \%$ of individuals with severe SARS-CoV-2 infections also develop neurological and psychiatric complaints. In rare cases, the occurrence of autoimmune encephalitis has been reported after SARS-CoV-2 infection. In this systematic review, we have identified eight SARS-CoV-2-associated cases of anti-NMDA receptor encephalitis. All had cerebrospinal fluid antibodies against the NMDA receptor and a recent onset of working memory deficits, altered mental status, or psychiatric symptoms, such as confusion, agitation, auditory hallucination, catatonia and speech dysfunction. All patients received high-dose steroid and immunoglobulin therapeutics and conditions improved in each case. These findings suggest that clinical attention should be paid to warning signs of autoimmune encephalitis in severe COVID-19 cases. If characteristic features of autoimmune encephalitis are present, autoantibody diagnostics should be performed and confirmed cases should be treated with immunotherapy to minimize neurological impairments.
\end{abstract}

Keywords: SARS-CoV-2, COVID-19, Inflammation, Autoimmune encephalitis, NMDA receptor, Corticosteroid, Immunomodulatory agent

\section{Introduction}

The COVID-19 pandemic caused by the SARS-CoV-2 virus has now affected more than $2 \%$ of the world population with over 185 million cases and 4 million deaths [1]. In addition to effects on health and mortality, the spread of the virus across the globe as well as governmental responses to the pandemic have had dire effects on human contacts and global economies and cases of anxiety and depression have increased in parallel, even in the non-infected population [2]. More directly, one study showed that approximately $30 \%$ of those infected by the virus and who experienced a severe course of the

\footnotetext{
*Correspondence: johann.steiner@med.ovgu.de

${ }^{1}$ Laboratory of Translational Psychiatry, Department of Psychiatry

and Psychotherapy, Otto-Von-Guericke-University Magdeburg, Leipziger

Str. 44, 39120 Magdeburg, Germany

Full list of author information is available at the end of the article
}

disease also developed psychological complaints, such as post-traumatic stress disorder (PTSD) [3]. Another study showed that of 125 severe cases registered as part of the CoroNerve study with neurological and psychiatric presentations of COVID-19 infection, 39 (31\%) presented with altered mental status and $23(18 \%)$ of these fulfilled the clinical case definitions for psychiatric disorders including new-onset psychosis, neurocognitive syndrome and affective disorder [4].

Yapici-Eser and colleagues recently described a potential pathomechanism based on molecular mimicry that may contribute to development of COVID-19-associated neuropsychiatric symptoms [5]. Structural similarities between the $N$-methyl-D-aspartic acid receptor (NMDAR) GluN1 (synonym NR1) and GluN2a (synonym NR2a) subunits with the SARS-CoV-2 nonstructural protein 8 (NSP8) and 9 (NSP9), respectively, may induce original author(s) and the source, provide a link to the Creative Commons licence, and indicate if changes were made. The images or other third party material in this article are included in the article's Creative Commons licence, unless indicated otherwise in a credit line to the material. If material is not included in the article's Creative Commons licence and your intended use is not permitted by statutory regulation or exceeds the permitted use, you will need to obtain permission directly from the copyright holder. To view a copy of this licence, visit http://creativecommons.org/licenses/by/4.0/. The Creative Commons Public Domain Dedication waiver (http://creativeco mmons.org/publicdomain/zero/1.0/) applies to the data made available in this article, unless otherwise stated in a credit line to the data. 
immune-mediated cross-reactivity to the NMDAR. These proteins are essential for replication of the virus and can interact directly with glutamate receptors of the NMDA and metabotropic families, leading to changes in membrane resting-state and action potentials [5]. Molecular mimicry may lead to generation of immunoglobulin G (IgG) antibodies against the NMDAR after SARS-CoV-2 infections. Anti-NMDAR encephalitis, mediated by IgG antibodies to the GluN1 subunit, is a common form of autoimmune encephalitis characterized by presentation of neurological and psychosis-like symptoms [6]. In this disease, antibodies bind to the NMDAR, induce crosslinking and receptors are subsequently internalized and thus are no longer available for excitatory glutamatergic transmission [6]. Viral diseases have been identified as potential triggers. For instance, anti-NMDAR-encephalitides can occur as a secondary disease after infection with viruses such as herpes simplex 1 or varicella zoster [7]. Moreover, past influenza A or B infections were identified as predisposing factors for NMDAR autoantibody seropositivity [8]. Accordingly, we hypothesized that SARS-CoV-2 might similarly induce anti-NMDAR encephalitis as a direct consequence of infection or secondarily through subsequent activation of autoimmune processes. Viruses such as SARS-CoV-2 hijack the cellular machinery of the host cell in order to reproduce themselves. In the process, their mimicry of key motifs of host proteins can lead to disruption of vital cellular functions, activate inflammation pathways and alter the immune response [9].

Here, we aimed to identify published cases of antiNMDAR encephalitis with concurrent neuropsychiatric symptoms temporally associated with SARS-CoV-2 infections.

\section{Methods}

We searched the PubMed and Google Scholar databases using the search terms "NMDA encephalitis" or "NMDAR encephalitis" or "NMDA receptor encephalitis" and "SARS-CoV-2" or "COVID-19" to identify relevant cases. The last search was performed on September 20th 2021. Preferred Reporting Items for Systematic Reviews and Meta-Analyses (PRISMA, http://prisma-statement. org) were applied. The process for selecting studies (identification, screening, eligibility and inclusion in the systematic review) is reported in a flow diagram in Fig. 1. Studies were checked for eligibility and selected by two persons (VV and PCG). Initially, many more papers were identified in Google Scholar than in PubMed. However, most of these articles did not meet the search criteria as, on close inspection, they were comments on already published articles, meta-analyses, etc. For the final evaluation, only case reports or case series in original reports published in peer-reviewed international journals were considered. Articles in languages other than English or German were translated with the help of DeepL Translator (https://www.deepl.com). Finally, only 7 English [10-16] and one Spanish article [11] met the search criteria. The quality of the identified case reports was checked according to the CARE Case Report Guidelines (2013 CARE Checklist: www.care-statement.org) as summarized in Additional file 1: Table S1. Cases were included if they were positive for SARS-CoV-2 in nasopharyngeal swab or blood or cerebrospinal fluid (CSF) tests and suffered from anti-NMDAR encephalitis as defined by Graus et al. [17]. We extracted information regarding patient age and medical history, reason for hospitalization, respiratory symptoms, neuropsychiatric symptoms, blood-based biomarkers, magnetic resonance imaging (MRI) of the brain, CSF biomarkers and electroencephalography (EEG) readings, as well as therapies attempted and clinical outcomes, as available.

\section{Results}

We identified eight case reports in which anti-NMDAR encephalitis (characteristic disease symptoms and detection of NMDAR antibodies in CSF) occurred with a time delay of 3 days to 3 weeks after the manifestation of COVID-19 disease (Table 1). Four of the identified 8 patients developed symptoms with a time delay of less than one week. The age of the patients ranged from 23 months to 53 years and consisted of four males and four females. All patients had positive GluN1 antibodies in the CSF. Only patients 1-4 showed pronounced respiratory symptoms including low $\mathrm{O}_{2}$ saturation, bilateral milk glass opacities on chest $\mathrm{x}$-ray and patchy bibasilar consolidation on chest computed tomography (CT) imaging [10-12], and severe hypoxemia with bilateral infiltrations [13]. Patients 2, 3 and 5-8 developed epileptic seizures during the course [11, 12, 14-16, 18]. All patients had psychiatric or neurological symptoms, manifested as a disturbance of consciousness, delirium, psychosis or catatonia. Patients 1 and 5-8 exhibited motor failures or dysarthria [10,14-16, 18]. Cases 4 and 8 had increased levels of C-reactive protein (CRP) and patients 3,4 and 8 had decreased lymphocytes [12, 13, 16]. Increased interleukin-6 (IL-6) levels were found in blood and CSF in one subject (patient 5) [14] and only in CSF in another (patient 1) [10]. Patients 1-6 had elevated lymphocytes in CSF [10-15]. Cases 3 and 8 were SARS-CoV-2 positive in the CSF $[12,16]$. Patient 2 had ovarian teratoma as an associated malignancy and potential alternative trigger of disease [11]. EEG recordings from patients 1, 4, 5 and 8 showed a slowdown, and one of these (patient 5) showed an extreme delta brush-like pattern, typical for the diagnosis $[10,13,14,16]$. In five 

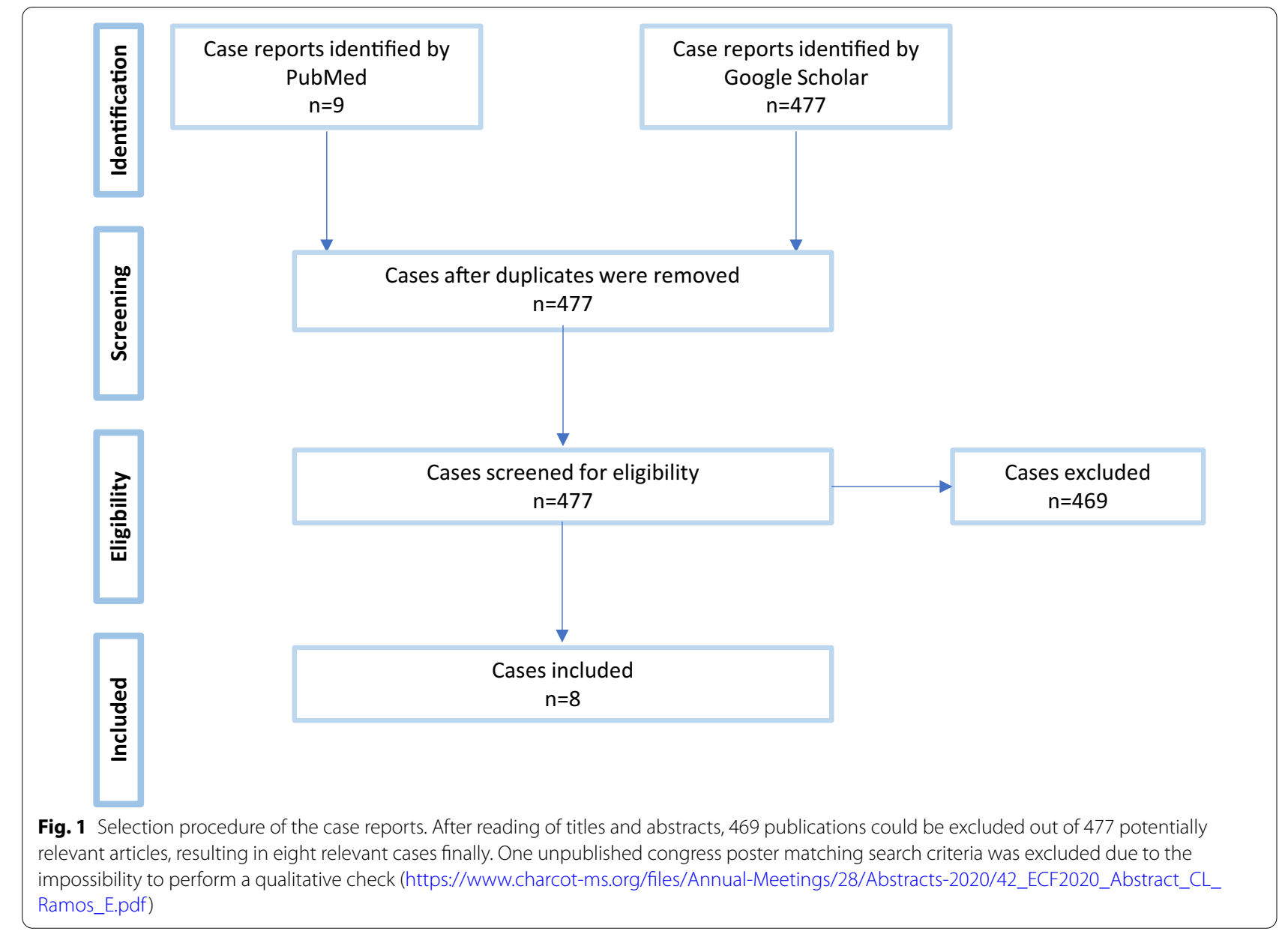

patients, imaging examinations of the brain appeared normal but patient 4 showed hyperintensities on fluidattenuated inversion recovery (FLAIR) images in left amygdala, left anterior putamen with negligible effects in the right amygdala [13]. It should be noted that in this latter case, an initial SARS-CoV-2 swab PCR test was negative, raising the possibility that the infection may have been hospital-acquired. Each patient received guidelinecompliant steroid therapy with intravenous immunoglobulins, patients 5 and 8 underwent plasmapheresis [14, $16]$, and conditions improved in all cases.

\section{Discussion}

This is the first systematic review to demonstrate a potential link between SARS-CoV-2 infection and secondary occurrence of anti-NMDAR encephalitis presenting with characteristic neuropsychiatric disorders. It is possible that mimicry of non-structural proteins of the SARSCoV-2 virus with NMDA receptor subunit epitopes may have been the underlying cause of the autoimmune response against brain NMDA receptors (Fig. 2). However, further studies are required to pinpoint the precise mechanism of how such effects of the virus lead to central nervous system (CNS) autoimmunity.

IL-6 and Th17 cells appear to be involved in the pathophysiology of COVID-19-associated neuropsychiatric symptoms [19, 20] (Fig. 2). IL-6 is a key proinflammatory cytokine that can alter neuronal and glial activity and induce axonal degeneration [21, 22]. Moreover, IL-6 levels correlate with the severity of disease course in COVID-19 [23]. IL-6-dependent Th17 activation and differentiation appears to be essential for neutrophil granulocyte migration [23]. Consequently, the proinflammatory cytokine IL-17 produced by Th17 CD4+ T cells may disrupt the blood-brain barrier (BBB) and contribute to the strong association of Sars-Cov-2 with a variety of different secondary autoimmune diseases (e.g. systemic lupus erythematosus, Guillain-Barré syndrome, antiphospholipid syndrome, large vessel vasculitis and thrombosis, psoriasis, inflammatory arthritis, autoimmune thyroiditis, type-I-diabetes) and circulating autoantibodies (e.g., anti-nuclear antibodies/ANA, anticardiolipin/aCL antibodies, cytoplasmic and perinuclear anti-neutrophil cytoplasmic antibodies/cANCA and 
Table 1 Summary of COVID-19 cases identified involving anti-NMDAR encephalitis with neuropsychiatric symptoms

Patient 1 Panariello et al., 2020 [10] Reason for hospitalization Respiratory (other) symptoms Neuropsychiatric symptoms

Blood test

CSF examination

EEG

Therapy

Course

Patient 2 Alvarez Bravo and Ramio, 2020 [11]

Reason for hospitalization

Respiratory (other) symptoms

Neuropsychiatric symptoms

Blood test

CSF examination

EEG

Therapy

Course

Patient 3 Allahyari et al., 2021 [12]

Reason for hospitalization Respiratory (other) symptoms

Neuropsychiatric symptoms

Blood test

CSF examination

EEG

Therapy

Course

Patient 4 McHattie et al., 2021 [13]

Reason for hospitalization

Respiratory (other) symptoms Neuropsychiatric symptoms
Male (23 years-old). History drug abuse

Psychomotor agitation, anxiety, formal thought disorder, persecutory delusions and auditory hallucinations and global insomnia. SARS-CoV-2 positive nasopharyngeal swab RT-PCR testing

Fever, drop in $\mathrm{O}_{2}$ saturation, chest $X$-ray: bilateral milk glass opacities, chest $\mathrm{CT}$ : patchy bibasilar consolidation

Confusion, disorganization of speech, thought/behaviour, auditory hallucinations and insomnia. Week 2: mutistic/non-responsive. Week-3: dysphagia, dyskinesia, autonomic instability, fluctuations in body temperature, blood pressure, pulse and respiratory rate

IL-6 not mentioned, no lymphopenia at anti-NMDAR encephalitis diagnosis, hyponatremia

SARS-CoV-2 negative. IL-6 elevated, NMDAR-antibodies positive. Virological and microbiological diagnostics negative. Elevated red and white cells

Theta activity, unstable, non-reactive to visual stimuli

Seizure prophylaxis. No symptom improvement with antipsychotics. COVID-19 therapy with hydroxychloroquine and darunavir/cobicistat. Antibiotic prophylactic therapy. After anti-NMDAR encephalitis diagnosis, dexamethasone and intravenous immunoglobulin

Clinical symptoms improved

testing

Behavioral changes

Fever, pneumonia, thrombosis of the left iliac vein, and bilateral pulmonary embolism attributed to SARS-CoV-2 infection

(Ovarian teratoma)

Psychomotor agitation, paranoid ideation, dysarthria with dysprosody, and visual hallucinations, focal and generalised seizures

SARS-CoV-2 positive

Cells count and protein elevated. SARS-CoV-2 negative, NMDAR antibodies positive. Virological and microbiological diagnostics negative

Epileptic discharges in the left frontotemporal region

After anti-NMDAR encephalitis diagnosis, 5 days of methylprednisolone and immunoglobulins administered

Hypoprosexia, emotional lability and memory disorder, Stabilised systemic and respiratory symptoms Female (18 years-old). No previous medical history. SARS-CoV-2 positive nasopharyngeal swab RT-PCR testing

Generalized tonic-clonic seizures

Fever, pneumonia, hypotonia, tachycardia, tachypnea, oxygen saturation of $90 \%$, bilateral pulmonary crackles in lower lung zones,

3-week history of mood change as depression and anhedonia accompanied by lack of concentration, generalized tonic-clonic seizures

Neutrophilia, lymphopenia, CRP normal

Cells count elevated. SARS-CoV-2 positive, NMDAR antibodies positive. Virological and microbiological diagnostics negative

Epileptic discharges in the left frontotemporal region

Seizure prophylaxis. COVID-19 therapy with Remdesivir, Lopinavir/Ritonavir, and Interferon b1a (Resigen). Antibiotic prophylactic therapy. After anti-NMDAR encephalitis diagnosis, methylprednisolone and intravenous immunoglobulin

After 2 months of hospitalization discharged with full recovery

Female (53 years-old). Ductal carcinoma of breast in remission. History of depression and psoriasis. Medications: sertraline, ciclosporin

2-week confusion, fever and myalgias. SARS-CoV-2 negative on admission, positive on day-14 in nasopharyngeal swab RT-PCR testing

Severe hypoxemia with $\mathrm{O} 2$ dependency. Chest $\mathrm{X}$-ray: bilateral infiltrations

Day-5: catatonic symptoms of severe echolalia, palilalia, perseverations and echopraxia. Speech highpitched and behavioural disinhibition. Left-side discrete hemiparesis, non-responsive to commands. Day-17: focal seizures, marked dysautonomia (increasingly hypotensive with bradycardia). Hyperkinetic movement disorder not present

CRP elevated with lymphopenia. NMDAR antibodies negative 
Table 1 (continued)

CSF examination

EEG

Therapy

Course

Patient 5 Monti et al., 2020 [14] Reason for hospitalization Respiratory (other) symptoms Neuropsychiatric symptoms

Blood test

CSF examination

EEG

Therapy

Course

Patient 6 Burr et al., 2021 [15]

Reason for hospitalization

Respiratory (other) symptoms Neuropsychiatric symptoms

Blood test

CSF examination

EEG

Therapy

Course

Patient 7 Sanchez-Morales et al., 2021 [18]

Reason for hospitalization Respiratory (other) symptoms Neuropsychiatric symptoms Blood test

CSF examination

EEG

Therapy

Course
SARS-CoV-2 negative. Leukocytes high. Low glucose and high protein. Virological and microbiological diagnostics negative. NMDAR antibodies positive

Slow activity on admission. No evidence of epileptiform discharges

Antiepileptic treatment. For suspected viral encephalitis, initial therapy with aciclovir and steroids. COVID-19 therapy with hydroxychloroquine, antibacterial and antifungal treatment. After anti-NMDAR encephalitis diagnosis, steroids, intravenous immunoglobulins and tocilizumab

Worsening symptoms with steroids. 1-month therapy: neuropsychiatric symptoms improved but persistence of left-side weakness. Cardiac MRI day 70: regression of signal changes. Brain MRI: atrophy of left amygdala and left hippocampus

Male (50 years-old). Moderate arterial hypertension

Acute psychiatric symptoms. SARS-CoV-2 positive nasopharyngeal swab RT-PCR testing

None. No diarrhoea. Fever present

Confabulations and delirium. Day-4: focal motor seizures with reduced consciousness, orofacial dyskinesia, automatisms. Sudden refractory status epilepticus

IL-6 elevated. No CRP elevation or leukocytosis

SARS-CoV-2 not mentioned. Third lumbar puncture: NMDAR antibodies positive, cell count and IL-6 elevated. Oligoclonal bands positive. Virological and microbiological diagnostics negative

Diffuse delta activity with extreme delta brush pattern.Anterior subcontinuous periodic theta activity

Antiepileptics and anaesthetics. COVID-19 therapy with hydroxychloroquine and lopinavir/ritonavir. After diagnosis of anti-NMDAR encephalitis: corticosteroids, immunoglobulins and plasmapheresis

4 months after symptom onset patient discharged in good condition with no neuropsychiatric symptoms

Female (23 months-old). Vaccinated. No previous diseases. Family history unremarkable

Fever, psychomotor agitation, sleep disturbances, constipation, decreased oral intake. SARS-CoV-2 positive nasopharyngeal swab RT-PCR testing

None. Fever, dehydration present

Agitation, poor sleep, mood swings, mutism, regular kicking/ flapping of extremities. Day-2: multiple epileptic seizures. Week 2: worsening encephalopathy with persistent hyperkinetic movements of extremities and head

CRP normal, NMDAR antibodies positive, IL-6 not mentioned

SARS-CoV-2 negative. Mild elevation of leukocytes. Oligoclonal bands negative. Virological and microbiological diagnostics negative. NMDAR antibodies positive. IL-6 not mentioned

Not mentioned

Antiepileptics. After anti-NMDAR encephalitis diagnosis, corticosteroid therapy for 5 days with no improvement, followed by intravenous immunoglobulin administration

Remission within one week after immunoglobulin therapy

Male (14 years-old). No previous medical history. SARS-CoV-2 positive nasopharyngeal swab RT-PCR testing

Behavioral changes and neurological symptoms

None

Altered behaviour and mental status, epileptic seizures, insomnia, orolingual dyskinesia

SARS-COV-2 negative

SARS-CoV-2 positive, NMDAR antibodies positive. Virological and microbiological diagnostics negative Not mentioned

After anti-NMDAR encephalitis diagnosis, methylprednisolone and immunoglobulins administered Complete remission of neurological impairment. Control of epilepsy. Persistence of psychiatric symptoms 
Table 1 (continued)

\begin{tabular}{|c|c|c|}
\hline \multirow[t]{9}{*}{ Patient 8} & Sarigecili et al., 2021 [16] & Male (7 years-old). Vaccinated. No previous diseases. No abnormal family history \\
\hline & Reason for hospitalization & Gait disorder. SARS-CoV-2 positive nasopharyngeal swab RT-PCR testing \\
\hline & Respiratory (other) symptoms & None. No headache, fever, or cold symptoms. Day 8: tachycardia \\
\hline & Neuropsychiatric symptoms & $\begin{array}{l}\text { Ataxia and broad-based gait with poor muscle reflexes. Day-2: somnolence and epileptic seizures. Day } \\
\text { 8: choreiform movements of extremities, tongue protrusion, bruxism, smacking, psychomotor agitation, } \\
\text { catatonia, echolalia }\end{array}$ \\
\hline & Blood test & CRP elevated, lymphopenia. IL-6 not mentioned \\
\hline & CSF examination & $\begin{array}{l}\text { No cells present. Oligoclonal bands negative. Virological and microbiological diagnostics negative. } \\
\text { NMDAR antibodies positive. IL-6 not mentioned }\end{array}$ \\
\hline & EEG & Encephalopathic pattern with disseminated delta waves \\
\hline & Therapy & $\begin{array}{l}\text { Antiepileptics after onset of seizures. Initial therapy with antibiotics/antivirals. After diagnosis of } \\
\text { anti-NMDAR encephalitis: plasmapheresis three times, corticosteroid } 7 \text { days, immunoglobulins } 5 \text { days } \\
\text { followed by corticosteroid again }\end{array}$ \\
\hline & Course & $\begin{array}{l}\text { Day 31: patient discharged walking but mildly ataxic with prednisolone and antiepileptic treatment. } \\
\text { Possibility of repeat immunoglobulin administration }\end{array}$ \\
\hline
\end{tabular}

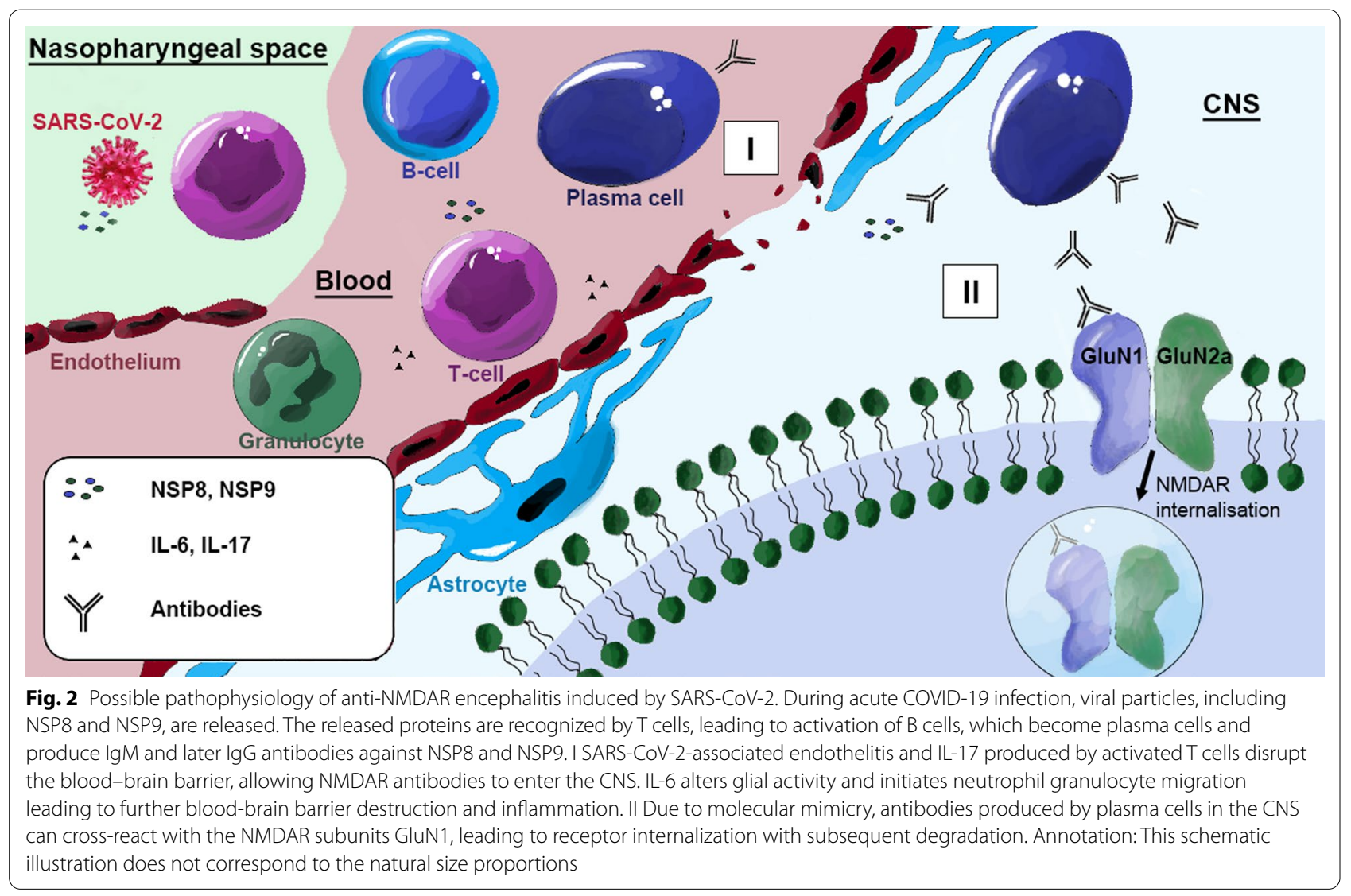

pANCA, antiprothrombin IgM) [24]. Accordingly, SarsCov-2 has been referred to as an "autoimmune virus" [24].
Alternatively, microvascular damage caused during the systemic inflammatory response to SARS-CoV-2 infection may promote development of encephalopathy in severe cases [21] (Fig. 2). Inflammatory mediators 
produced by the alveolar epithelium, macrophages, and leukocytes may contribute to endothelial inflammation, increased vascular permeability, edema, and increased turnover of coagulation factors. The increased vascular permeability can also cause disturbances of the microcirculation including BBB impairment [21]. This disruption of the $\mathrm{BBB}$ gives rise to another possible mechanism involving facilitated transfer of NMDAR-directed antibodies, into the CNS [25].

This study was limited by the small number of patients identified with SARS-CoV-2-related autoimmune antiNMDAR encephalitis. In addition, an aetiological role of SARS-CoV-2 in the generation of anti-NMDAR encephalitis is not definitive. In one of the cases an initial SARSCoV-2 swab was negative and the patient only tested positive after the onset of anti-NMDAR symptoms [13]. In another case, an ovarian teratoma was present which is a potential alternative trigger of anti-NMDAR encephalitis [11]. However, more carefully-characterised studies should become available as the pandemic progresses and eventually recedes. This is important as COVID-19 patients with severe infections might have a higher risk for developing neurological manifestations such as autoimmune encephalitis. This highlights the importance of appropriate screening of patients using serological and CSF assessment via cell-based-assays for antineuronal antibodies, as well as brain imaging and EEG recordings [26]. Potentially, one might also include biomarkers for glial or neuronal damage such as S100B, myelin basic protein, neurofilament light chain (NfL), or neuronspecific enolase to screen for brain alterations [27-29]. Furthermore, it is important to monitor patients for neurological warning signs of autoimmune encephalitis including seizures, personality and memory changes, psychotic symptoms, delusional thinking, headache, dizziness, catatonia and dyskinesias [17, 30, 31].

The eight cases of anti-NMDAR encephalitis detailed in this paper also calls attention to the increased risk associated with the sudden onset or intensification of neuropsychiatric symptoms. All of the autoimmune encephalitis patients described in this study improved following high-dose steroids and immunoglobulin therapy, thus highlighting the importance of early immunotherapy once the diagnosis of autoimmune encephalitis has been made.

\footnotetext{
Abbreviations

aCL: Anti-cardiolipin antibody; ANA: Anti-nuclear antibody; BBB: Blood-brain barrier; CANCA: Cytoplasmic anti-neutrophil cytoplasmic antibody; CD4+T cells: Cluster of differentiation 4 expressing lymphocytes matured in the thymus; CNS: Central nervous system; COVID-19: Coronavirus disease 2019; CRP: C-reactive protein; CSF: Cerebrospinal fluid; CT: Computed tomography; EEG: Electroencephalography; FLAIR: Fluid-attenuated inversion recovery; GluN1: N-Methyl-D-aspartic acid receptor subunit 1; GluN2a: N-Methyl-D-aspartic acid
}

receptor subunit 2a; IgG: Immunoglobulin G; IgM: Immunoglobulin M; IL: Interleukin; MRI: Magnetic resonance imaging; NfL: Neurofilament light chain; NMDA: N-Methyl-D-aspartic acid; NMDAR: N-Methyl-D-aspartic acid receptor; NR1: N-Methyl-D-aspartic acid receptor subunit 1; NR2a: N-Methyl-D-aspartic acid receptor subunit 2a; NSP8: Nonstructural protein 8; NSP9: Nonstructural protein $9 ; \mathrm{O}_{2}$ : Oxygen; pANCA: Perinuclear anti-neutrophil cytoplasmic antibody; PTSD: Post-traumatic stress disorder; PubMed: Search engine accessing primarily the MEDLINE (Medical Literature Analysis and Retrieval System Online) database of references and abstracts on life sciences and biomedical topics, maintained by the United States National Library of Medicine (NLM) at the National Institutes of Health; SARS-CoV-2: Severe acute respiratory syndrome coronavirus type 2; Th17 cells: Thelper cells producing interleukin 17.

\section{Supplementary Information}

The online version contains supplementary material available at https://doi. org/10.1186/s12974-021-02293-x.

Below is the link to the electronic supplementary material.Additional file 1. Table S1. The quality of the identified case reports has been checked according to the CARE Case Report Guidelines (www.care-state ment.org). For patient 7, this check was not feasible, because this case came from a retrospective original report: Sanchez-Morales et al., 2021 [18].

\section{Acknowledgements}

Not applicable.

\section{Authors' contributions}

HGB and JS defined the research of interest and were involved in topic selection. W and PCG carried out the literature search and created Table 1. W, PCG and JS wrote the first draft of the manuscript. HGB and CG reviewed the manuscript and made contributions for improvement. MLS contributed to the revision process with his expertise on the topic of systematic reviews and meta-analyses. All authors helped to revise the paper. All authors read and approved the final manuscript.

\section{Funding}

Open Access funding enabled and organized by Projekt DEAL. This work has been partly supported by European Research Area Network (ERA-NET) NEURON "Translational Biomarkers in Brain Disorders" (Project NicAb, grant no. 01EW2012).

Availability of data and materials

Not applicable.

\section{Declarations}

Ethics approval and consent to participate

Not applicable.

\section{Consent for publication}

Not applicable.

\section{Competing interests}

The authors declare that they have no competing interests.

\section{Author details}

${ }^{1}$ Laboratory of Translational Psychiatry, Department of Psychiatry and Psychotherapy, Otto-Von-Guericke-University Magdeburg, Leipziger Str. 44, 39120 Magdeburg, Germany. ${ }^{2}$ Laboratory of Neuroproteomics, Department of Biochemistry and Tissue Biology, University of Campinas (UNICAMP), Campinas, Brazil. ${ }^{3}$ Department of Neurology, Max Planck Institute for Human Cognitive and Brain Sciences, Clinic for Cognitive Neurology, University Hospital Leipzig, Leipzig, Germany. ${ }^{4}$ Section Translational Neuroimmunology, Department of Neurology, Jena University Hospital, Jena, Germany. ${ }^{5}$ German Center for Mental Health (DZP), Center for Intervention and Research On Adaptive and Maladaptive Brain Circuits Underlying, Mental Health (C-I-R-C), 
Jena-Magdeburg-Halle, Germany. ${ }^{6}$ Center for Behavioral Brain Sciences, Magdeburg, Germany.

Received: 14 July 2021 Accepted: 8 October 2021

Published online: 28 October 2021

\section{References}

1. COVID-19 Dashboard-by the Center for Systems Science and Engineering (CSSE) at Johns Hopkins University. COVID-19 Map. https://coron avirus.jhu.edu/map.html.

2. Kunzler AM, Rothke N, Gunthner L, Stoffers-Winterling J, Tuscher O, Coenen M, Rehfuess E, Schwarzer G, Binder H, Schmucker C, et al. Mental burden and its risk and protective factors during the early phase of the SARS-CoV-2 pandemic: systematic review and meta-analyses. Glob Health. 2021;17:34.

3. Janiri D, Carfi A, Kotzalidis GD, Bernabei R, Landi F, Sani G. Gemelli against C-P-ACSG: posttraumatic stress disorder in patients after severe COVID-19 infection. JAMA Psychiat. 2021;78:567-9.

4. Varatharaj A, Thomas N, Ellul MA, Davies NWS, Pollak TA, Tenorio EL, Sultan M, Easton A, Breen G, Zandi M, et al. Neurological and neuropsychiatric complications of COVID-19 in 153 patients: a UK-wide surveillance study. Lancet Psychiatry. 2020;7:875-82.

5. Yapici-Eser H, Koroglu YE, Oztop-Cakmak O, Keskin O, Gursoy A, GursoyOzdemir Y. Neuropsychiatric symptoms of COVID-19 explained by SARS-CoV-2 proteins' mimicry of human protein interactions. Front Hum Neurosci. 2021;15: 656313.

6. Kayser MS, Dalmau J. Anti-NMDA receptor encephalitis, autoimmunity, and psychosis. Schizophr Res. 2016;176(1):36-40. https://doi.org/10. 1016/j.schres.2014.10.007.

7. Prüss H. Autoantibodies in neurological disease. Nat Rev Immunol. 2021 https://doi.org/10.1038/s41577-021-00543-w.

8. Hammer C, Stepniak B, Schneider A, Papiol S, Tantra M, Begemann M, Siren AL, Pardo LA, Sperling S, Mohd Jofrry S, et al. Neuropsychiatric disease relevance of circulating anti-NMDA receptor autoantibodies depends on blood-brain barrier integrity. Mol Psychiatry. 2014;19:1143-9.

9. Joubert B, Dalmau J. The role of infections in autoimmune encephalitides. Rev Neurol (Paris). 2019;175:420-6.

10. Panariello A, Bassetti R, Radice A, Rossotti R, Puoti M, Corradin M, Moreno M, Percudani M. Anti-NMDA receptor encephalitis in a psychiatric COVID19 patient: a case report. Brain Behav Immun. 2020;87:179-81.

11. Alvarez Bravo G, Ramio ITL. Anti-NMDA receptor encephalitis secondary to SARS-CoV-2 infection. Neurologia (Engl Ed). 2020;35:699-700.

12. Allahyari F, Hosseinzadeh R, Nejad JH, Heiat M, Ranjbar R. A case report of simultaneous autoimmune and COVID-19 encephalitis. J Neurovirol. 2021;27:504-6.

13. McHattie AW, Coebergh J, Khan F, Morgante F. Palilalia as a prominent feature of anti-NMDA receptor encephalitis in a woman with COVID-19. J Neurol. 2021. https://doi.org/10.1007/s00415-021-10542-5.

14. Monti G, Giovannini G, Marudi A, Bedin R, Melegari A, Simone AM, Santangelo M, Pignatti A, Bertellini E, Trenti T, Meletti S. Anti-NMDA receptor encephalitis presenting as new onset refractory status epilepticus in COVID-19. Seizure. 2020;81:18-20.

15. Burr T, Barton C, Doll E, Lakhotia A, Sweeney M. N-Methyl-d-aspartate receptor encephalitis associated with COVID-19 infection in a toddler. Pediatr Neurol. 2021;114:75-6.

16. Sarigecili E, Arslan I, Ucar HK, Celik U: Pediatric anti-NMDA receptor encephalitis associated with COVID-19. Childs Nerv Syst. 2021;1-4. https://doi.org/10.1007/s00381-021-05155-2.
17. Graus F, Titulaer MJ, Balu R, Benseler S, Bien CG, Cellucci T, Cortese I, Dale RC, Gelfand JM, Geschwind M, et al. A clinical approach to diagnosis of autoimmune encephalitis. Lancet Neurol. 2016;15:391-404.

18. Sanchez-Morales AE, Urrutia-Osorio M, Camacho-Mendoza E, RosalesPedraza G, Davila-Maldonado L, Gonzalez-Duarte A, Herrera-Mora P, Ruiz-Garcia M. Neurological manifestations temporally associated with SARS-CoV-2 infection in pediatric patients in Mexico. Childs Nerv Syst. 2021;37(7):2305-12. https://doi.org/10.1007/s00381-021-05104-z.

19. Wu D, Yang XO. TH17 responses in cytokine storm of COVID-19: an emerging target of JAK2 inhibitor Fedratinib. J Microbiol Immunol Infect. 2020;53:368-70.

20. Parackova Z, Bloomfield M, Klocperk A, Sediva A. Neutrophils mediate Th17 promotion in COVID-19 patients. J Leukoc Biol. 2021;109:73-6.

21. Pacheco-Herrero M, Soto-Rojas LO, Harrington CR, Flores-Martinez YM, Villegas-Rojas MM, Leon-Aguilar AM, Martinez-Gomez PA, CampaCordoba BB, Apatiga-Perez R, Corniel-Taveras CN, et al. Elucidating the neuropathologic mechanisms of SARS-CoV-2 infection. Front Neurol. 2021;12: 660087.

22. Cazzolla AP, Lovero R, Lo Muzio L, Testa NF, Schirinzi A, Palmieri G, Pozzessere P, Procacci V, Di Comite M, Ciavarella D, et al. Taste and smell disorders in COVID-19 patients: role of interleukin-6. ACS Chem Neurosci. 2020;11:2774-81.

23. Gubernatorova EO, Gorshkova EA, Polinova Al, Drutskaya MS. IL-6: relevance for immunopathology of SARS-CoV-2. Cytokine Growth Factor Rev. 2020;53:13-24.

24. Halpert G, Shoenfeld Y. SARS-CoV-2, the autoimmune virus. Autoimmun Rev. 2020;19: 102695.

25. Ehrenreich $\mathrm{H}$. Autoantibodies against the N-Methyl-d-Aspartate receptor subunit NR1: untangling apparent inconsistencies for clinical practice. Front Immunol. 2017;8:181.

26. Vasilevska V, Guest PC, Schlaaff K, Incesoy El, Prüss H, Steiner J. Potential cross-links of inflammation with schizophreniform and affective symptoms: A review and outlook on autoimmune encephalitis and COVID-19. Front Psychiatry. 2021;12:729868.

27. Streitbürger DP, Arelin K, Kratzsch J, Thiery J, Steiner J, Villringer A, Mueller K, Schroeter ML. Validating serum S100B and neuron-specific enolase as biomarkers for the human brain - a combined serum, gene expression and MRI study. PLoS ONE. 2012;7: e43284.

28. Schroeter ML, Steiner J. Elevated serum levels of the glial marker protein S100B are not specific for schizophrenia or mood disorders. Mol Psychiatry. 2009;14:235-7.

29. Khalil M, Teunissen CE, Otto M, Piehl F, Sormani MP, Gattringer T, Barro C, Kappos L, Comabella M, Fazekas F, et al. Neurofilaments as biomarkers in neurological disorders. Nat Rev Neurol. 2018;14:577-89.

30. Pollak TA, Lennox BR, Muller S, Benros ME, Pruss H, Tebartz van Elst L, Klein H, Steiner J, Frodl T, Bogerts B, et al. Autoimmune psychosis: an international consensus on an approach to the diagnosis and management of psychosis of suspected autoimmune origin. Lancet Psychiatry. 2020;7:93-108

31. Dalmau J, Gleichman AJ, Hughes EG, Rossi JE, Peng X, Lai M, Dessain SK, Rosenfeld MR, Balice-Gordon R, Lynch DR. Anti-NMDA-receptor encephalitis: case series and analysis of the effects of antibodies. Lancet Neurol. 2008;7:1091-8.

\section{Publisher's Note}

Springer Nature remains neutral with regard to jurisdictional claims in published maps and institutional affiliations. 